\title{
Using Social Determinants Screening/Mapping Tools to Identify Needs and Resources for Student-Run Free Clinic Patients
}

\author{
Akanksha Dadlani'; Cooper T. Johnson'; Rebecca Fischbein ${ }^{1}$; Stacey L. Gardner- \\ Buckshaw ${ }^{1}$; Amy Lee ${ }^{1}$; John M. Boltri ${ }^{1}$ \\ ${ }^{1}$ Department of Family and Community Medicine, Northeast Ohio Medical University, Rootstown, $\mathrm{OH}$ \\ Corresponding Author: Akanksha Dadlani, 4209 State Route 44, PO Box 95, Rootstown, OH 44272, (925) 518-6394, adadlani@neomed.edu \\ Submitted November 21, 2020 Accepted April 6, 2021 Published June 21, 2021 https://doi.org/10.18061/ojph.v4i1.8073
}

\section{ABSTRACT}

Background: Social determinants of health have been well accepted as contributing to health outcomes. They are a vital aspect of health care delivery and must be a consideration, especially among free clinic populations. Social determinants of health have also become a required element of medical school curricula. The Student Outreach to Area Residents Student-Run Free Clinic based out of Northeast Ohio Medical University piloted a student-led program that implemented social determinants of health screening and community resource referral as a part of integrated health care delivery for all its patients.

Methods: We described the development of a screening tool, protocol, and creation of community resource referral materials. We also described the tracking of patient-reported needs and mapping of location and accessibility of community resources. One hundred patients were surveyed through convenience sampling, and results were used for program improvement.

Results/Conclusion: After collecting and analyzing survey results, it was found that the 2 most frequently requested determinants were mental health and utilities services, and the most available community resource was emergency food services. We also mapped these results by zip code and found gaps between need and distribution of services. We demonstrated the utility of mapping to identify points of improvement for the future. We also provided lessons learned related to effective social determinants of health screening, community resource referral, and overall program implementation in student-run free clinics. We further explained the benefits of including similar student-led programs as a way for students to gain practical experience related to social determinants of health.

Keywords: Social determinants of health; Mapping; Student-run free clinic

\section{INTRODUCTION}

Social determinants of health (SDOH), or an individual's social situation related to life and work such as income, education, access to health care, and housing, have a strong influence on health. ${ }^{1}$ While these SDOH have a large impact on health status, they are often ignored in medicine: $60 \%$ of the preventable deaths in the United States (US) have been shown to be caused by modifiable behaviors and exposures in the community, yet more than $95 \%$ of the money spent on health care in the US is for direct medical services. ${ }^{2}$
In recognition of the critical impact of SDOH, in primary care settings increasing attention is paid to screening for SDOH such as inequalities/variances in income, education, employment, selfreport of disease, social environment, and housing.3,4 Benefits of screening and referral include providing whole-person care, increasing cost effectiveness, and reducing utilization of services downstream. ${ }^{3}$

Despite increased interest and attention regarding SDOH screening and referral in primary care, many providers report concerns 
about conducting screens with patients. ${ }^{5,6}$ Structural barriers to SDOH screening include lack of reimbursement for screening ${ }^{7}$ as well as insufficient time to implement this activity with patients. ${ }^{8}$ Other provider concerns are related to training such as lack of skills and knowledge to perform screens and uncertainty of how to adequately perform referrals.5,6 Because this gap in medical education has been identified, efforts have been made to alleviate it in future physician training. Current medical school curricula require education related to SDOH. Specifically, the Liaison Committee on Medical Education (LCME) accreditation standards 7.5 and 7.6 require education related to "societal problems," and "recognition of the impact of disparities in health care on medically underserved populations and potential solutions to eliminate health care disparities," respectively. ${ }^{9}$

One way to enhance student exposure to and experience with SDOH screening and referral is through integration of these services into student-run free clinics. For more than a decade, the number of student-run free clinics has been increasing across the US.10 Early research has shown that medical students and patients believe free clinics are valuable for education and care quality. ${ }^{11}$ Student-run free clinics provide free, high quality services to vulnerable patients, most of whom are uninsured or underinsured. ${ }^{11-13}$ Given the demographics of patients at student-run free clinics and the need to incorporate SDOH in medical education, both patients and students could benefit from enhanced SDOH screening and referral in student-run free clinics. For example, education programs at free clinics led by medical students have facilitated clinical improvements among patients with diabetes who also experience economic and social disadvantage. ${ }^{14}$ Additionally, there is evidence that early-career first-year medical students develop comfort with diverse patients under the mentorship of fourth-year medical students. ${ }^{15}$ And physicians who receive training in community-based and underserved settings are more likely to practice in similar settings, such as health centers. ${ }^{16}$

Despite the potential benefits of incorporating SDOH screening student-run free clinics, the integration of such services in student-run free clinics may be lacking. ${ }^{17}$ Only limited published research exists regarding the implementation of SDOH screening and referral services into student-run free clinic settings and how to assess the match between patient needs and the availability of local services surrounding student-run free clinics. ${ }^{18}$ Consequently, the primary goal of this work is to describe an SDOH screening and referral process designed and implemented by medical students at a rural student-run free clinic. Specifically, this work describes: 1) the process used to develop and implement the screening protocol, 2) screening results from a convenience sample of 100 patients, and 3) how screening results were analyzed using mapping software and needs assessment of community resources to match needs to referral resources. We conclude with lessons learned and how similar processes can assist with both health professions student education as well as patients' needs.

\section{METHODS}

\section{Setting}

The Student Outreach to Area Residents (SOAR) student-run free clinic is based at Northeast Ohio Medical University (NEOMED) in a rural portion of Portage County, where $10 \%$ of the population is uninsured. Additionally, in Portage County, 45\% of adults reported cost as an issue with their health coverage, $8 \%$ opted out of some coverage because they could not afford it, and $17 \%$ of adults did not fill prescriptions from their doctor. ${ }^{19}$ The US Census Bureau does not explicitly define the term "rural." Rootstown, Ohio, is categorized as an urban cluster on rural land. On the US Census Bureau website, however, Rootstown, Ohio, is mapped as a rural area. ${ }^{20}$

Founded in 2017, SOAR offers primary care, chronic disease management, physical exams, basic labs, prescription for medications, dietician services, diabetes education, and medication therapy management. Since its inception, SOAR has had over 920 patient visits with roughly 280 unique patients and approximately 700 follow-up visits. The clinic operated 3 Saturdays a month from 2019 to winter 2020. Roughly 12 patients are seen on an average clinic date. Twenty-five students work an average clinic day with 355 medicine and pharmacy student volunteers, 29 physicians, and 16 pharmacists working at the clinic every year. Eighty percent of the patients are native to Portage County, with $73 \%$ of the patients uninsured and $89 \%$ living below the 200 percent federal poverty level line. Based on informal observation of patients' needs, it was determined that patients could benefit from more formal SDOH screening and referral process.

\section{Community, participant characteristics, recruitment}

Starting in September 2019, attempts were made to screen every new and returning SOAR patient. One hundred patients completed the screening form on their own while in the waiting room. The only inclusion criterion was that participants had to be patients of the clinic. No compensation was provided. The results were reviewed by student volunteers who then did the following: 1) entered patient's room with completed survey and requested permission to discuss results with patient, 2) upon consent, reviewed with the patient potential matching resources including hours of operation, location and services, and 3) wrote down and provided information about the suggested resource(s) for the patient to take home.

To launch screening and referral in SOAR, a community resource team of 22 medical student volunteers were selected and trained to administer screening and provide referrals. Training included didactic orientation, observation of the authors performing screenings and referrals, and practice performing activities while being observed by the authors.

\section{Procedures/Program description}

The lead author conducted a literature review of existing SDOH screening tools and toolkits. Google Scholar and PubMed were the 
primary resources used for the literature review. Keywords included, "social determinants of health," "student run free clinic," "PDSA," "program implementation," "community asset inventory," "community asset," and "rural." The goal was to see if other similar programs existed and, if they did, how they were implemented into the clinic's workflow. The organization Health Leads was selected as a model for our program as it has a preexisting infrastructure for a similar screening survey and workflow. ${ }^{21}$ The Health Leads tool requires patients to complete a screening tool so their provider could identify adverse social determinants of health to be addressed by multidisciplinary care teams. The screening tool includes food insecurity, housing, utility needs, financial resources strain, transportation, exposure to violence, demographic information with options to add childcare, education, employment, health behaviors, social support and mental health as determined by the health system and/or program initiative. ${ }^{22}$

The Health Leads screening tool was adapted for use in Portage County. The decision to include specific screening items was guided by a review of the resources available in the community. A starting point for this process included examination of preexisting student-developed lists of community resources. Community resources were defined as any local resource that would provide aid for the given categories at either no cost, prorated cost, or sliding scale. Other factors taken into account include hours of operation, availability, and eligibility criteria. Each resource was individually audited for hours of operation, location, payment, and services offered. This process resulted in the creation of an organized list of resources labeled based on categories matching the screening tool (eg, food, housing, transport). Upon finalization of the list of available resources, items from the corresponding Health Leads categories were selected. For this analysis, services that provided childcare include psychiatric services, counseling, case management, social skills groups, daycare, and development services. Pet care includes providing food, low-cost clinics, neutering, and adoption programs. Utility services assist with paying bills and connecting clients with attorneys. Clothing services include gently used clothing at no cost, clothing for interviews, and clothing for children.

\section{Measures/Outcomes}

The measures for this study are common SOAR patient needs. If a patient indicated "yes" to a particular need, we classified that as a need, and it was recorded in a deidentified, secure document. The current project uses this survey's data and compares it to deidentified patient zip code data/demographic information. These data were combined with publicly available data related to community resources and bus lines in Portage County, provided by the Portage Area Transit Authority (PARTA).

\section{Statistical analysis}

Analyses included descriptive statistics of the common patient needs and maps using ArcGIS Online. ${ }^{19}$ Esri ArcGIS Online was used to create maps for this analysis. Patient home zip codes were spatially joined to zip code shape files from the US Census Bureau. A choropleth map was created to depict areas with high and low density of patients compared to areas with high and low density of services. A second choropleth map of patient zip codes was created which added addresses of resources. Additionally, local bus routes were overlaid on the addresses of organizations to highlight possible public transportation barriers. General transportation barriers were defined as any difficulty in getting to the clinic as perceived by the patient. This can include, but is not limited to, lack of access to a vehicle, lack of access to a bus route, or inability to drive a vehicle.

\section{RESULTS}

One hundred patients were screened using the SDOH instrument between September 2019 and December 2019. Patients were mostly female (57\%), White (90\%), and with an average age of approximately 48 years. Most patients were uninsured (80\%) with the remainder covered by a private insurer $(12 \%)$ and Medicare (7\%); no patient was billed. Medicaid is not as prevalent in this population because patients with Medicaid have $\$ 0$ copay to see primary care physicians. They also have $\$ 0$ copay for emergency and urgent care visits. Because they have access to medical care otherwise, they are not our primary source of patients.

Responses were tallied across the 100 participants and patientidentified needs were compared to corresponding community resources (Table 1).

Table 1. Frequency of Reported Patient Needs $(n=100)$ and Available Community Resources $(n=55)$

\begin{tabular}{|lll|}
\hline Patient need & $\begin{array}{l}\text { Frequency of patients reporting a need } \\
(\mathbf{n}=\mathbf{1 0 0} \text { patients)* }\end{array}$ & $\begin{array}{l}\text { Count of available community resources } \\
\mathbf{( n = 5 5} \text { resources) }\end{array}$ \\
Mental health & $18(18 \%)$ & $9(16 \%)$ \\
Utilities & $18(18 \%)$ & $4(7 \%)$ \\
Transportation & $15(15 \%)$ & $3(5 \%)$ \\
Emergency food & $11(11 \%)$ & $16(29 \%)$ \\
Substance abuse & $11(11 \%)$ & $5(9 \%)$ \\
Legal & $9(9 \%)$ & $4(7 \%)$ \\
Children care & $5(5 \%)$ & $3(5 \%)$ \\
Clothing & $5(5 \%)$ & $2(4 \%)$ \\
Housing & $4(4 \%)$ & $6(11 \%)$ \\
Pet care & $3(3 \%)$ & $2(4 \%)$ \\
Hygiene & $2(2 \%)$ & $1(2 \%)$ \\
*Patients could report more than 1 need, therefore sum of total needs is greater than 100
\end{tabular}

*Patients could report more than 1 need, therefore sum of total needs is greater than 100 . 
Twenty-one percent of patients reported having more than 1 need, for a mean of 4 needs. Among all patients, the most frequently reported needs were mental health and utilities (both 18\%) followed by transportation (15\%). Only $2 \%$ and $3 \%$ of patient needs were identified to be hygiene and pet needs, respectively. The most commonly available community resource within and near Portage County is emergency food assistance (29\%) followed by mental health resources (16\%) and then housing (11\%). Despite being frequently reported needs among patients, resources in the community for utilities and transportation were limited $(7 \%$ and $5 \%$, respectively).

To better understand the proximity of community resources relative to the SOAR patient population, patient zip codes were mapped against the location of community resources; specifically, we plotted the proportion of patients to community resources per zip code (Figure 1).

Areas in Figure 1 that are dark pink have a high number of patients and services. Dark grey areas have a low number of patients and a high number of services. Areas that are peach have a high number of patients and a low number of services.
In Figure 2, patient population is depicted with darker shades corresponding to a high number of patients, and lighter zip codes indicate few patients. The median number of patients per zip code was 20 .

Each type of service is represented by colored dots. Location and clustering of services are useful for comparing patient needs against resource availability. For example, all emergency food services are red dots and are located in 6 unique zip codes. These resources are not located in town centers but are often located at faith-based organizations which are more widespread. Mental health services, the second most requested resource, are primarily located in 2 areas (Akron, Ohio, and Kent, Ohio). This illustrates a potential gap in services since the majority of mental health services are concentrated in Akron, Ohio, which is approximately a 25 -minute drive from the zip code with the highest number of patients. While the local bus routes connect many of the available resources, many of the peripheral zip codes are not serviced by the local bus authority. The SOAR facility is not serviced by PARTA routes during SOAR's operating hours. Depending on the route, PARTA operates Monday through Friday or Monday through Sat-

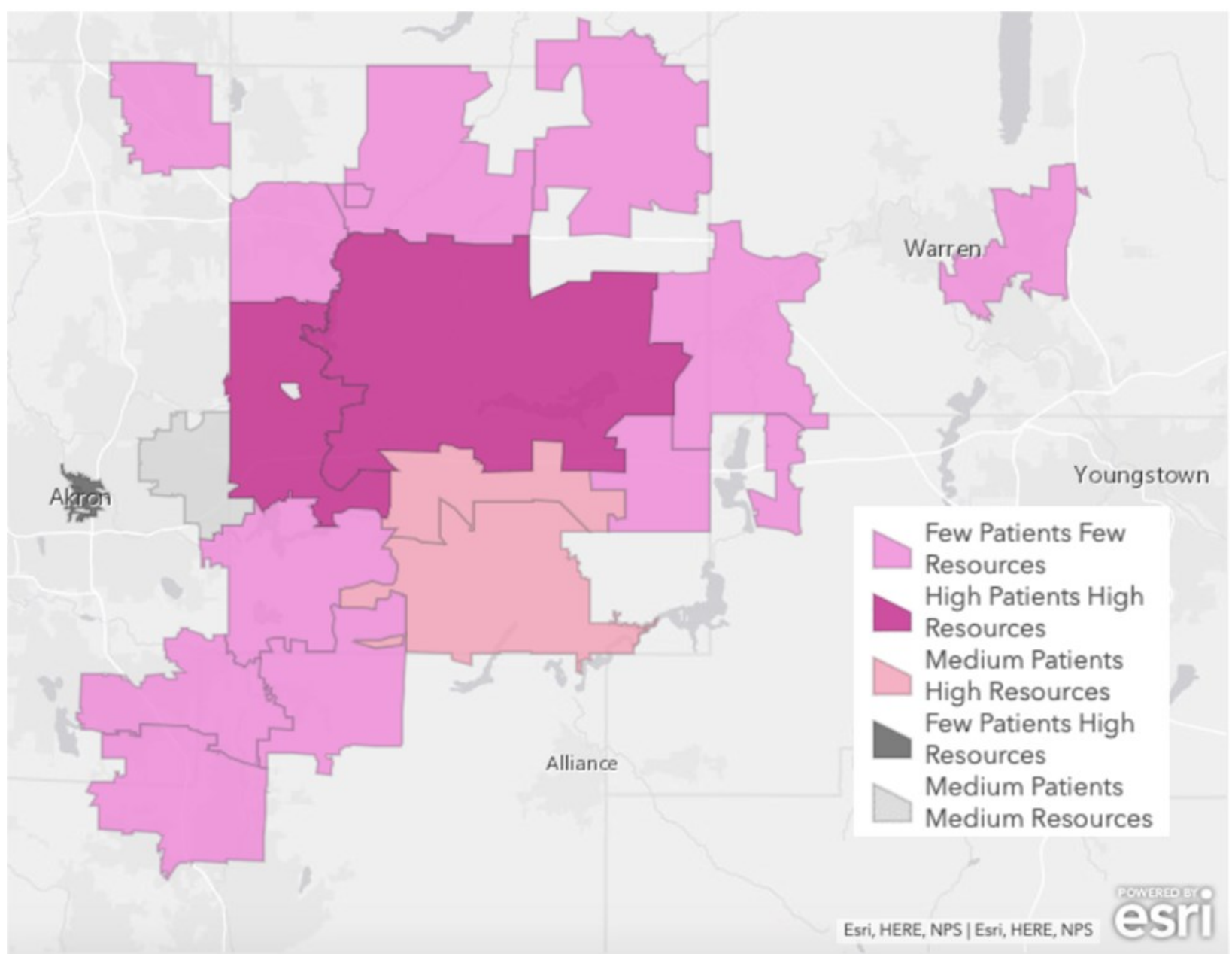

The light pink zip codes represent areas that have few patients and few community resources. The dark pink areas represent areas that have a high density of patients and resources. 


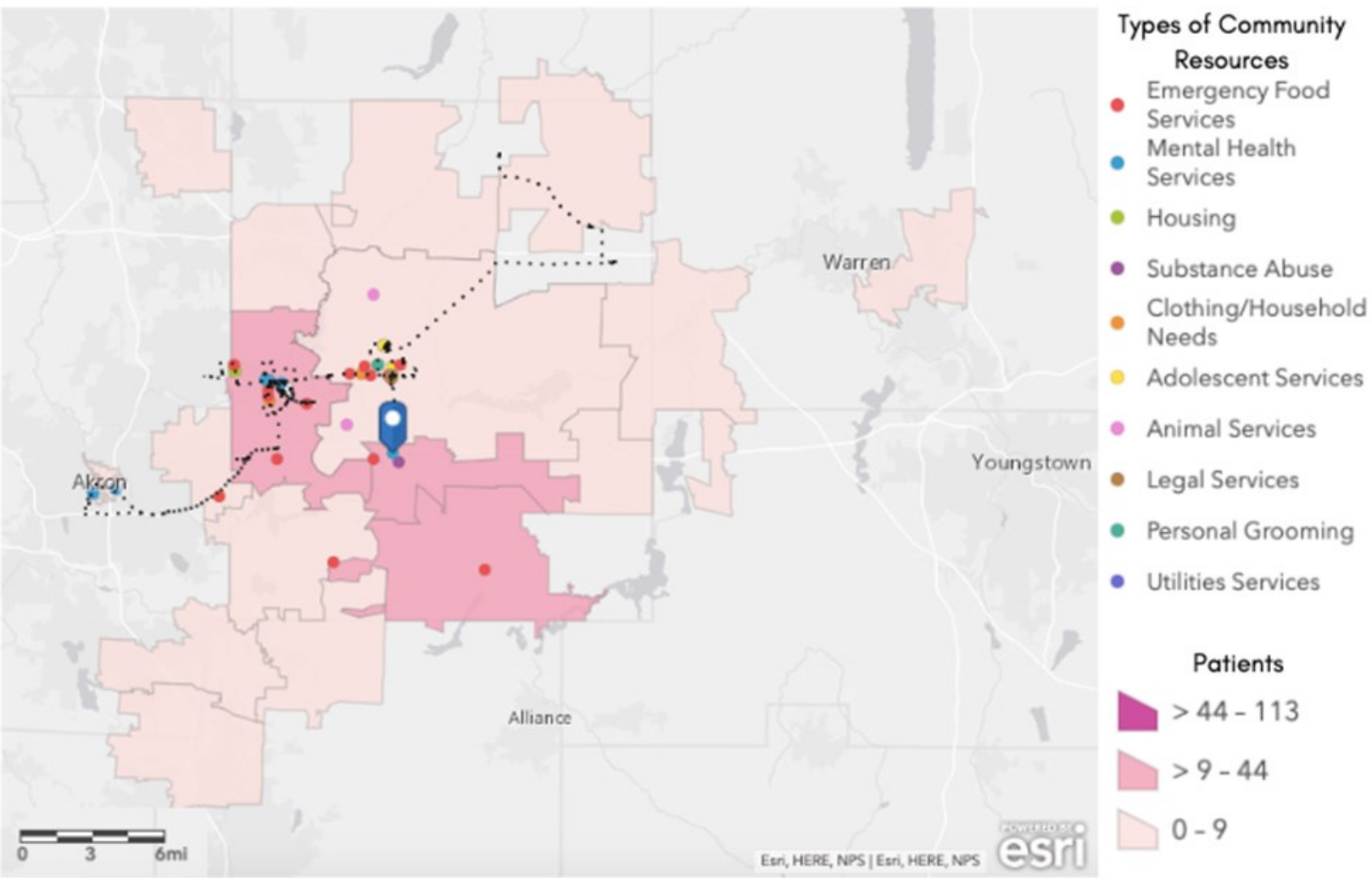

The blue point in the center of the map represents the SOAR Clinic location. The individual type of services are identified by the colored dots with each color representing a different type of service. The dark lines correspond to bus routes across Summit County and Portage County.

\section{Figure 2. Comparison of SOAR Patient Zip Codes to Location of Specific Community Resources and Bus Routes}

urday. While the major bus route has 9 stops and circulates about every 30 minutes, the closest bus stop to SOAR stops approximately 4 miles away, with no additional local public transportation.

\section{DISCUSSION}

In this study we describe the development of a community free clinic-specific screening tool, an implementation protocol, and the creation of community resource referral materials. However, resources are useless if they cannot be accessed by patients in need. Therefore, we visually presented patient-reported needs of 100 patients utilizing the free clinic for primary care and mapped the location and accessibility of community resources. This project provides an example and lessons learned when depicting SDOH screening and referral integration into a student-run free clinic. We have described the information that can be gathered by conducting social needs screening among patients at a student-run free clinic and then using tools like mapping software to compare that information against resources available in the community. We have demonstrated how this activity can reveal a disparity in terms of diversity and geographic distribution of resources.

Beyond our own clinic, we hope that this data collection method can serve as a model for other student-run free clinics conducting needs assessment and gap analysis. Because SOAR is in a rural area, community resources are sparse and there are many barriers in connecting patients to appropriate resources. While limited research exists about conducting needs analysis and mapping in urban settings, ${ }^{18}$ we were unable to find literature on these topics related to rural areas.

In only 2 zip codes, there are a high number of both services and patients. Figure 2 also details the service area the SOAR Clinic covers; the farthest zip code is roughly 30 miles away, confirming that more resources are available for patients who live in urban/ suburban areas while less is available for our many patients who live in rural locations. ${ }^{23}$ This lack of distribution could be a challenge for some patients who lack adequate transportation, especially given that many of the rural areas where our patients reside are not served by the local bus authority.

Our analysis clarifies the need for mental health and substance abuse services for many SOAR patients. In response, our clinic recently expanded to include a behavioral health consultant who can conduct, and guide SOAR students with, behavioral health screening, brief intervention, and referral to treatment. This project also demonstrates the prevalence and significance of social needs in this free clinic population. Of the patients surveyed, 21\% had more than 1 need, with an average of 4 needs. 


\section{Limitations and lessons learned}

Our implementation of SDOH screening and referral in the SOAR clinic was not without limitations. First, when choosing which SDOH to include in the screening, more formal data collection and inclusion of patients as part of the development of the screening tool using a community-based participatory method would have been highly beneficial. This would have likely avoided inclusion of some of the less critical needs (eg, pet and hygiene) as well as inclusion of women's health care, a missed need. Additionally, we were limited in the needs we could include in the screener. For example, based on anecdotal reports from patients and staff, we suspected our patients had unmet dental needs; yet Portage County had no available dental resources for patients without insurance at the time of screening. Therefore, we did not include this need in our screener as screening without provision of appropriate resources could be considered wasteful and unethical.8,24 However, after the collection of this pilot data, we found a dentist willing to take patients with little or no insurance and included this need in our screening.

Further, despite the physical geographic distance, some services could be provided remotely, with the potential to reduce geographic disparities. We did not include in our screening whether patients would or would be able to remotely access services for these needs (eg, by telephone or virtually). Further, we did not break down our scan of available community resources in this manner. However, with the onset of coronavirus disease 2019 (COVID-19), the ability for patients to have remote access to services has become more relevant than ever. ${ }^{25}$ In fact, the SOAR Clinic quickly adapted to provide telephone-based and virtual services for patients. Moving forward, future revisions of screeners and community asset inventory will include information related to remote access for services.

While the community resource team did not address remote access for services, it has updated the community asset inventory to include current and relevant COVID-19 resources. Correspondingly, community resources should always be added and subtracted based on availability and quality of the local resources. Teams should periodically check on resources to determine if all the provided information is still valid and applicable. Establishing a strong community resource network involves time and effort including online research, cold calling, and seeking out partners within the community that can help navigate resources.

Finally, we also recognize that even if only a few resources are available to address a high demand need, this does not necessarily indicate patients will be underserved by those resources. For example, there are only 3 community resources for transportation, but it is the third most indicated concern for patients at the clinic. However, we tend to recommend only a single transportation resource because it works well for patients and is a high-quality service. In other words, quantity cannot be equated with quality.
For potential future projects, it would be beneficial to include other methods of assessing of resource distribution. For example, as indicated above, adding an element which objectively captures quality of the resources would help when determining what referrals to provide and which services to consider adding internally to the clinic. Similarly, following up with patients over time would permit us to gauge the efficacy of resources and whether patients are benefiting from our recommendations. This long-term tracking of SDOH, use of resources, and interaction with the patient education team will be facilitated by the clinic's recent transformation to electronic health records (EHR). The SDOH screen will become part of the patient record in the EHR. Eventually, we would like for it to be added to the patient's problem list to ensure that it is regularly revisited and addressed. Additionally, comparing the home address of patients (rather than just zip codes) to the addresses of social services would allow for a more detailed location-based examination of needs. For example, there may be an area that has a high need for emergency food services that does not have a food pantry nearby. Currently, we do not know if that level of disparity exists. Also importantly, as indicated above, future screening and referral will take into account patients' interest and ability to remotely access services as well as the ability of community resources to provide remote services.

Based on mapping analysis, there are large disparities in how patients and social services are distributed. Spatial segregation and clustering of poverty are both present issues in our community. While we do not have a mobile clinic, we have recently started doing telemedicine visits. Using telemedicine comes with unique barriers, but we hope that some of the disparities can be addressed with this new method of patient contact. There is no current plan to do a "clinic on wheels" or expand the clinic to have multiple locations, though it is a consideration given the difficulty to access. There are local "clinics on wheels" services that we have contacted, and we are waiting to hear back from them.

\section{PUBLIC HEALTH IMPLICATIONS}

Nearly one-fourth of our patients surveyed are facing multiple needs, highlighting the importance of providing SDOH screening and referral for our patient population. The significance of SDOH in health care delivery and student medical education is clear.1,9 The next step in providing necessary care for patients involves implementation of SDOH screening and referral programs, ${ }^{3}$ as well as continuous improvement of this process. This project has not only added value to the quality of care provided at SOAR, but also allowed students to experience provision of health care in a way that is often beyond the scope of traditional medical school curriculum. As SDOH becomes a more integral part of health care delivery and medical school curriculum, ${ }^{9}$ projects like these provide students with an opportunity to learn by doing; students gained exposure to the significance of social determinants of health in a clinical setting. ${ }^{13,15}$ 


\section{ACKNOWLEDGMENTS}

The authors received no financial support for the research, authorship, and/or publication of this article.

Conflict of interest statement. The authors declare that they have no conflict of interest.

Compliance with ethical standards. The NEOMED University institutional review board determined that the project did not constitute human subjects research

\section{REFERENCES}

1. WHO Commission on Social Determinants of Health, World Health Organization, eds. Closing the Gap in a Generation: Health Equity through Action on the Social Determinants of Health: Commission on Social Determinants of Health Final Report. World Health Organization, Commission on Social Determinants of Health; 2008.

2. Alley DE, Asomugha CN, Conway PH, Sanghavi DM. Accountable health communities-addressing social needs through Medicare and Medicaid. $N$ Engl J Med. 2016;374(1):8-11.

https://doi.org/10.1056/NEJMp1512532

3. Andermann A. Screening for social determinants of health in clinical care: moving from the margins to the mainstream. Public Health Rev. 2018;39:19.

https://doi.org/10.1186/s40985-018-0094-7

4. Cadzow RB, Servoss TJ, Fox CH. The health status of patients of a student-run free medical clinic in inner-city Buffalo, NY. J Am Board Fam Med. 2007;20(6):572-580.

https://doi.org/10.3122/jabfm.2007.06.070036

5. British Medical Association. Social Determinants of Health: What Can Doctors Do? British Medical Association; 6455. Accessed March 66, 6464. https://www.paho.org/hq/dmdocuments/2012/Social-DeterminantsDoctors-Eng.pdf

6. Fenton M. Health Care's Blind Side: The Overlooked Connection between Social Needs and Good Health. The Robert Wood Johnson Foundation Accessed March 22, 2020.

https://sirenetwork.ucsf.edu/tools-resources/resources/health-caresblind-side-overlooked-connection-between-social-needs-and

7. Chisolm DJ, Brook DL, Applegate MS, Kelleher KJ. Social determinants of health priorities of state Medicaid programs. BMC Health Serv Res. 2019;19(1):167. https://doi.org/10.1186/s12913-019-3977-5

8. O'Gurek DT, Henke C. A Practical Approach to Screening for Social Determinants of Health. Fam Pract Manag. 2018;25(3):7-12.

9. Liaison Committee on Medical Education. Functions and Structure of a Medical School: Standards for Accreditation of Medical Education Programs Leading to the MD Degree. Association of American Medical Colleges and American Medical Association; 2019. Accessed March 22, 2020.

https://lcme.org/publications/

10. Smith S, Thomas R, Cruz M, Griggs R, Moscato B, Ferrara A. Presence and Characteristics of Student-Run Free Clinics in Medical Schools. JAMA. 2014;312(22):2407. https://doi.org/10.1001/jama.2014.16066

11. Simpson SA, Long JA. Medical Student-Run Health Clinics: Important Contributors to Patient Care and Medical Education. J Gen Intern Med. 2007;22(3):352-356.

https://doi.org/10.1007/s11606-006-0073-4
12. Swartz MK. The contributions of student-run free clinics. JPediatr Health Care. 2012;26(6):397.

https://doi.org/10.1016/j.pedhc.2012.07.015

13. Smith SD, Johnson ML, Rodriguez N, Moutier C, Beck E. Medical student perceptions of the educational value of a student-run free clinic. Fam Med. 2012;44(9):646-649.

14. Gorrindo P, Peltz A, Ladner TR, et al. Medical students as health educators at a student-run free clinic: improving the clinical outcomes of diabetic patients. Acad Med. 2014;89(4):625-631. https://doi.org/10.1097/ACM.0000000000000164

15. Choudhury N, Khanwalkar A, Kraninger J, Vohra A, Jones K, Reddy S. Peer mentorship in student-run free clinics: the impact on preclinical education. Fam Med. 2014;46(3):204-208.

16. Phillips RL, Petterson S, Bazemore A. Do residents who train in safety net settings return for practice?: Acad Med. 2013;88(12):1934-1940. https://doi.org/10.1097/ACM.0000000000000025

17. Warren S, Puryear E, Chapman M, Barnett TM, White LS. The role of social work in free healthcare clinics and student-run clinics. Soc Work Health Care. 2017;56(10):884-896. https://doi.org/10.1080/00981389.2017.1371097

18. Lefer TB, Anderson MR, Fornari A, Lambert A, Fletcher J, Baquero M. Using Google Earth as an innovative tool for community mapping. Public Health Rep. 2008;123(4):474-480. https://doi.org/10.1177/003335490812300408

19. Portage County Community Health Partners. 2019 Portage County Community Health Needs Assessment;; 2019. Accessed June 5, 2020. https://www.hcno.org/wp-content/uploads/2019/12/FINAL-2019. Portage-County-CHNA-12-6-19.pdf

20. United States Census Bureau. How does the census bureau define rural? Rural America. Accessed March 30, 2021.

https://mtgis-portal.geo.census.gov/arcgis/apps/MapSeries/ index.html? appid=49cd4bc9c8eb444ab51218c1d5001ef6\#: :text=The\% 20Census\%20Bureau\%20defines\%20rural,rural\%20based\%20on\% 20this\%20definition

21. Health Leads. Social Needs Screening Toolkit. Published online 2018. Accessed March 23, 2020.

https://healthleadsusa.org/resources/the-health-leads-screeningtoolkit/

22. National Alliance to Impact Social Determinants of Health. Screening Tools and Tool Kits. Helpful Screening Tools and Tool Kits. Published 2019. Accessed February 25, 2021.

https://www.nasdoh.org/screening-tools-and-tool-kits/

23. Douthit N, Kiv S, Dwolatzky T, Biswas S. Exposing some important barriers to health care access in the rural USA. Public Health. 2015;129 (6):611-620. https://doi.org/10.1016/j.puhe.2015.04.001

24. Perrin EC. Ethical questions about screening. J Dev Behav Pediatr. 1998;19(5):350-352. https://doi.org/10.1097/00004703-199810000-00006

25. Kronenfeld JP, Penedo FJ. Novel coronavirus (COVID-19): telemedicine and remote care delivery in a time of medical crisis, implementation, and challenges. Transl Behav Med. Published online October 24, 2020:ibaa105.

https://doi.org/10.1093/tbm/ibaa105 